\title{
The Paradigms of Uncertainty
}

\author{
by René Passet*
}

In his paper " Reflections on Uncertainty ", W. A. Weisskopf establishes a connection between two stages of economics and two paradigms taken from physics : one coming from Newton's mechanics, the other based on Heisenberg's principle of uncertainty. In this way he places economics at its rightful level which is multidisciplinary.

The connection he makes between two important federating systems of physics and economic theory is doubly justified :

- by the fact that economics is often explicitly built on models which come either from the sciences of matter or from the sciences of living things;

- and by the fact that any system of thought which, consciously or otherwise, is steeped in the intellectual atmosphere of its age, can only be fully apprehended in relation to this atmosphere.

According to Weisskopf, the transition from the paradigm of Newton to that of Heisenberg reflects the shift from a mechanistic-deterministic vision to a vision which opens onto undeterminancy and uncertainty. I would like here to state that I agree with this general line of reasoning and that $I$ feel it is necessary to extend it to considerations of another group of paradigms, which are common to living things and inanimate objects : the paradigms of thermodynamics and of information, which take undeterminancy right to the heart of reality and extend the debate to the forces of evolution which drive economic systems.

\section{From Newton to Heisenberg : from a mechanistic and deterministic paradigm to a vision which opens onto undeterminancy and uncertainty.}

The Newtonian paradigm had a definite influence on economic theory. Several writers make specific reference to it :

- Jevons defines economics as " the mechanics of utility and of individual interest";

- for Walras " the system of the economic universe is revealed in all its greatness and complexity: a system both vast and simple which resembles in sheer beauty the astronomic universe" ;

* Professor of economics at the University of Paris (Sorbonne). This text has been translated from French, by Rosalind Greenstein. 
- according to Rueff: "dynamics is that part of mechanics which studies motion and the way it is related to the influences which cause it ... economic dynamics has recourse to the same method".

General equilibrium is nothing more than cosmic order born out of universal attraction and transposed to economics.

From Newton's mechanistic system (where the "démon de Laplace" who knows everything is master) Weisskopf quite rightly dissociates a paradigm of uncertainty born, according to him, from Heisenberg's principle. Although, as far as I know, few economists have explicitly acknowledged their debt to this paradigm, I think, as Weisskopf suggests, that a whole trend of thought uses it and takes this uncertainty directly into account (Knight), or introduces it into economic analysis through the strategy of agents who:

- either have a certain freedom of movement in markets where transparency and fluidity are no longer considered perfect (imperfect or monopolistic competition) ;

- or cease beeing micro-agents and reach those dimensions which allow them to influence the market (monopoly, oligopoly...).

However it must be recognised that some fundamentally liberal, neoclassical and explicitly Newtonian writers have been able to take possession of this paradigm, turn it upside down and interpret it differently. Thus, at the end of his life J. Rueff, in a rather moving book "Les Dieux et les Rois", asked himself whether the development of quantum theory did not undermine the Newtonian foundations on which he had based his economics. On the contrary he ended up confirming his position.

According to him :

- the principle of uncertainty represents, at the heart of the particle, the symbol and germ of that particular individual liberty on which he built his economics;

- this uncertainty, which has been verified at elemental level, in no way contradicts the existence of a determinism and of laws governing statistical populations : the laws of probability allow us to reconcile the "liberty" of the particle with, for example, the existence of laws on the behaviour of gases; it is the same thing in economics where the liberty of individual agents does not contradict the fact that global supply and demand may obey certain laws; in this way the market is a melting pot where the meeting of the masses results in an equilibrium.

All in all Rueff finds in Heisenberg's work (what he was looking for, of course...) a confirmation of his mechanistic position. On this point he evokes Louis de Broglie who wrote: "When we rise up to the macroscopic world the discontinuous character of individual phenomena disappears, submerged so to speak in statistics. Complementary and in a way irreconcilable descriptions, in terms of corpuscle and wave, in terms of location in space and time, in terms of dynamic state, come together and melt in the harmonious moulds of classical physics. (Matière et Lumière quoted in "Les Dieux et les Rois ", p. 69).

This "salvaging" is possible, I feel, because the indetermination connected with Heisenberg's principle is only at the level of micro-elements. At the global level of macro-phenomena, it changes the sense of determinism which ceases to be mechanistic and becomes statistic (with all the important consequences that this implies, in particular 
for the reversibility of time) : but it does not destroy it. We can go as far as to ask ourselves the true meaning of uncertainty in Heisenberg's work. Does it come from the interference between the observer and that which is observed in low energy zones (in the field of long waves, where the precision of observation requires waves which are as short as possible and therefore energies which are likely to distort the behaviour of the object)? Is it due to the inherent constraints of the mathematical "operators" which are used (noncommutability of matrix multiplication) and which can only reveal one aspect of reality - velocity or position - at a time and not both together? In short, is it due to the nature of things or due to the knowledge we may have of them ? As an economist I have not the right to come down one way or the other, in subjects not my own ; I can only observe that the question remains open and ask myself whether, in the final instance, the principle of undeterminancy really does lead to a true paradigm of uncertainty.

Weisskopf quite rightly relates the transition from one paradigm to another to historical factors, particularly those concerning the structural transformation of economic systems. Paradoxically, however, neither of the two paradigms proposed seems to allow this transformation to be taken into account. It gives rise to the paradigms but is not explained by them.

Indeed, any apprehension of history seems totally excluded on the basis of a Newtonian analysis, where matter untiringly repeats the same movements, where time eventless - leads nowhere and where general economic equilibrium does not lead to any dynamics. It does not seem any more probable in economics "à la Heisenberg ", in which the market, in view of its statistical determinism, finally fulfilling its regulation function, does not seem to be a carrier of those desequilibria which are necessary if transformation is to take place.

The experience of the transformation of the world through the action of human beings - which generates new social ways of thinking - seems to be historically connected to the advent of the use of fire: whereas the use of mechanical forces (falling water, wind, resilience...) in no way altered the environment, the coal which is consumed today and transformed into smoke and ashes, will no longer exist tomorrow.

Here we enter the realm of thermodynamics.

Thermodynamics breaks completely with the Newtonian system. It started in 1811 , the year the Baron Jean-Joseph Fourier, Prefect of Isère, won the Académie prize for his essay on the propagation of heat through solids. Carnot established it as a science. As Prigogine and Stengers wrote (" La Nouvelle Alliance ", Seuil 1979, p. 119) : " henceforth a physical theory exists, which is both mathematically as rigorous as the mechanical laws of motion and totally foreign to a Newtonian world (...), on the other hand it is a law which is as general as Newton's laws (...). Every body has a mass and interacts gravitationally with every other body in the Universe ; but every body is also capable of receiving, accumulating and transmitting heat and, as such, is the centre of all the processes related to the accumulation and the propagation of heat." We know that this science spread successively from closed systems (the so-called "classical" linear equilibrium thermodynamics of Carnot, Clausius, Thomson, Boltzmann) to open systems which are close to an equilibrium state (Onsager, Schrödinger), then to open systems which are far from an equilibrium state (non-linear thermodynamics of dissipative structures : Prigogine). 


\section{The paradigms of thermodynamics : uncertainty at the heart of things}

Insofar as the intermediate phase of evolution summarised above is essentially transitional, we shall only dwell on its two extreme forms.

- In classical linear equilibrium thermodynamics, when a system obeys the principle of entropy, it destructures itself and the difference in potential which allowed it to transform its potential energy into kinetic energy is progressively reduced; this is in accordance with the laws of chance, as Boltzmann demonstrated. Probability acts here as an "attractor": for instance, when a gas which is concentrated in one of two communicating compartments of a closed system spreads throughout the two compartments, this occurs in a random way, as the particles crash against the walls of the compartment; when uniform distribution is reached (entropy), it is once again according to the laws of probability that the movements of the particles compensate each other and the system remains homogeneous.

- In non-linear nonequilibrium thermodynamics, an increase in energy leads to morphogenesis. The system goes through "critical" points, from which it structures itself : when the flow of a river reaches a certain speed a whirlpool appears; when an oily liquid is heated from underneath, hexagonal structures form on the surface (Benard whirls)... The important thing is that the "divergences" which appear at the critical points and through which matter structures itself occur at random. The general outline of the phenomenon is determined, but the detail of its morphology and the precise order of events leading up to it are not.

We cannot say in advance which of the directions opened up by the critical point the transformation will take. When all is said and done, all reality is but the revelation of one of the possibilities.

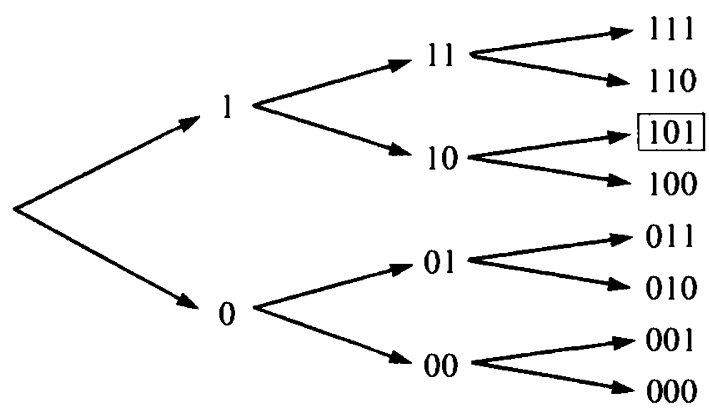

But, contrary to the implications of Newtonian determinism (where the knowledge of the position and the law of motion of a star at a given moment enabled one to determine its position at any moment in the past or future), this possibility cannot be identified in advance. In the tree branches shown above where 3 binary choices open up $2^{3}=8$ possible forms of structuring, system 101, the one which actually occurred, is the result of a 
progression where chance plays an important role and which, therefore, will not necessarily occur each time. Henceforth chance is to be found at the centre of the event and, as Prigogine and Stengers wrote: " the demon of Laplace is dead".

Two consequences, which are fundamental to economics, now impose themselves on us:

- the effect of the intrusion of chance is to orientate time and to make it irreversible : the world is going somewhere, in a determined direction, but in a non-specified manner; uncertainty forbids us from predicting with any accuracy what will be tomorrow and it is not sufficient to inverse the signs of a system of equations to know what was yesterday: chance does not undo in reverse order what it did with time ;

- the content of this time and the direction of this motion are different, depending on which thermodynamics are taken : time in classical thermodynamics is that of general degradation and breakdown; that of dissipative structures is that of creation and increasing complexity. In short, each of these models symbolises, in a remarkable way, one of these two contradictory yet complementary aspects of economic activity, in that, by transforming the world to adapt it to the demands of people, this one is at the same time an act of destruction and of creation.

\section{Classical thermodynamics : the economics of self-destructuring}

In my opinion the paradigm of classical thermodynamics lies at the root of the marxian view of history. In economic terms the parallel to the movement of the world towards entropy is the movement of self-destruction (and self-destructuring) which pulls the capitalist system along.

In actual fact the marxian system must be considered from two angles.

On the one hand the models of simple or enlarged reproduction, if taken to represent the relations that any system should respect if it is to maintain itself or to grow without any fundamental disequilibrium (which is impossible for the capitalist system, according to Marx and Engels), belong to a logic which is quite close to that of mechanics.

But on the other hand Marxism is also - maybe " above all " - a conception of evolution and of history, of the future history of capitalism. It is here, whatever the reservations Engels in particular may have had towards the second law of thermodynamics, that the latter seems to have deeply permeated the whole system of marxian thought. This analogy is not artificial : the two writers constantly refer to thermodynamics, particularly in their correspondence; this indicates that they were perfectly informed about the scientific developments of their age and that they wished to apply these teachings to the analysis of socio-economic phenomena.

Thus, in marxian thought (as in the first thermodynamics) we find that oriented time is moving towards a final situation, communist society, where - in the same way that a homogenised system is in a state of entropy - all class differences, all state structures, all contradictions which generate movement will have disappeared.

In the course of this evolution, the very driving forces which propel it are progressively depleted (in the same way that the progressive reduction of a difference in potential leads an energy system towards inertia). This depletion is manifested by the downward trend in the profit rate, the proletarisation movement, the depletion of the labour force 
and of the earth (the two pillars which, according to Marx, support the system), the exclusion from the productive apparatus of an ever-increasing section of the labour force - living capital, unique source of surplus value - to be replaced by machines, dead capital which cannot produce value.

Darwin, introduced here to explain the class struggle, remains a stranger in the house. The struggle for life does indeed govern the behaviour of the species, but nothing can explain their appearance in a physical universe of which science describes the progressive degradation and not the movement towards greater complexity which was able to engender life.

\section{Dissipative structures : the economy of complexification}

The paradigm of dissipative structures which answers this last question seems to define the broad outline of economics as it should be in our day and age.

It is interesting to note that this paradigm developed at a time when we were beginning to see more clearly the consequences, on the biosphere, of the logic of an economy which is both timeless and enclosed in its own values. A dissipative structure, on the contrary, is an open structure which can only maintain itself by being open. This calls for :

- An economic science which is open to life:

The thermodynamics of dissipative structures, in fact, renews the alliance between inanimate objects and living things. It highlights the way a movement towards greater complexity (which is essential for living things to emerge) can be created spontaneously, on the level of matter, under the impulse of energies which are sufficiently powerful to push a system away from the areas of Boltzmann's equilibrium thermodynamics.

This dovetails remarkably well with the works of Von Foerster and Atlan who show - in the field of information - how order and complexity can come out of erratic disturbance (" noise "). Life appears then to be an ineluctable phenomenon - and not, as Monod said, an accident - in our world.

All self-reproducing systems, be they individuals or societies, are dissipative structures, open to each other and only able to maintain themselves and to grow interdependently. This is particularly true of all economic systems (companies or nations, for instance), which only exist and develop by taking things out of the milieu into which they at the same time dispose of their waste.

It is therefore obvious, in an age of dwilling resources and of pollution, that the reproduction and development of the economy cannot be analysed independently of the human sphere and biosphere which sustain it. Our age calls for an economic science of the living.

- An economic science which is open to historical time:

The Newtonian paradigm describes a situation where the present flows mechanically from the past and contains all the future. The unexpected does not exist. Each and every moment contains all the history of humankind so that time can be eliminated. General equilibrium as built on this model, in the field of economics, is indeed a-temporal and can be repeated ad infinitum.

The intrusion of chance creates the event. Each occurrence represents, as we have seen, the materialisation of one of the possibilities, and not the one and only solution. 
We are in a universe - quite close to our everyday experience - where determinism and indeterminism find themselves closely entwined. Let us go back to our binary tree. The critical point confronts us with uncertainty : and it is here that the action of human beings (often minorities) can change the course of history.

But, once we have gone beyond this point, the fact of choosing one of the branches of the fork binds humankind in a determinism : for example option (I) implies, for the future, type-I situations and eliminates any solution beginning with $(0)$; in other words every choice that is made limits the range of possibilities. But this determinism is never absolute since we know that further critical points are waiting for us.

The result is that we can never understand a system unless we retrace the history of the events which engendered it. An economic science which wishes to renew contact with reality cannot afford to be a-temporal.

- An economic science which is open to values:

It is in enclosing itself in its own logic and with the pretension of subjugating the biosphere - by projecting itself as value and finality - that the economy contributes to the disturbance of natural mechanisms and is a threat to the living.

What the theory of dissipative structures teaches us is what can be called "the multiplicity of laws". Neither Newton nor Boltzmann nor Onsager are invalidated by Prigogine. Each of these writers remains valid in a well-defined field. Prigogine merely shows that the gradual distancing from areas of equilibrium thermodynamics reveals new forms of behaviour of matter and new mechanisms which can only be apprehended through specific systems of interpretation. Whereas before, the "temptation for unity" ended up in a dichotomous vision which was incapable of embracing both living things and inanimate objects, the multiplicity of laws allows a vision which reconciles the two.

It is the condemnation of all reductionism, in perfect agreement, may I add, with the systemic vision invoked by Weisskopf.

The role of the economy is not, therefore, to submit the Universe - and humankind - to its laws. It can only ensure its own survival by respecting the regulating mechanisms of the biosphere - the logic of which is totally alien to that of the market - which are so many constraints to be obeyed. And it can only find a justification as an ancillary science, in the service of human values.

In the face of these requirements orthodoxies harden and it is but slowly, too slowly, that economic science progresses along this triple path. No doubt it progresses more slowly than the evolution of events that W. A. Weisskopf showed us, so rightly, to be determining in the transformation of dominant paradigms. The economic paradigm that emerged from the thermodynamics of dissipative structures is not even in place and already we see taking shape in the shadows the broad outlines of an economy of the future which will give birth to new paradigms.

\section{The germination of a paradigm? Information and uncertainty in the very nature of things}

A certain mode of development based on energy and matter, taking the forms of concentration, comes up against its own limitations. Having been defined as a " complex- 
ifying growth ", development presupposes an accumulation of means, a diversification of activities and an exponential growth of its network of internal relations, which are necessary for its activities to fit together coherently. To put it clearly, systems devote the greater part of their resources to metabolic activities of structuring and functioning which increase to the detriment of their own performance.

The most highly developed economies, the behavioural patterns of which mark out the future, seem to be taking entirely new directions:

- as far as demand is concerned : the satisfaction, as a top priority, of vital organic needs (survival economies), then the satisfaction of needs for durable consumer goods (comfort economies), are followed by a period dominated by the appearance of a new kind of needs - leisure activities, culture, health - which concern essentially one's being and one's person;

- as far as supply is concerned : the driving forces of economic development are moving further and further away from the field of matter and energy and are polarising around the control of information (computers, telematics, microprocessors...) and the domestication of organic processes (biotechnologies); W. Kendrick has calculated that non-material investment (training, research, research-development) account for $50 \%$ of the increase in the global productivity of factors in the U.S.A. between 1948 and 1966, for $68 \%$ between 1966 and 1973 and for $100 \%$ between 1973 and 1978 (W. Kendrick in "Microelectronics, Productivity and Employment", OECD, 1981, p. 25); in computing nowadays $90 \%$ of expenditure goes on software and $10 \%$ on hardware, whereas 20 years ago the proportions were exactly the reverse.

Consequently the regulating mechanisms of our economies - the logic of which underlies all economic calculation - will apparently have to be totally revised :

- both in computing and in biotechnologies, given that most production costs concern research and equipment before the production process even begins, global costs hardly vary any longer with the volume of production; the notion of marginal cost loses all meaning ;

- since the efficiency of the combination of productive factors is, right from the start, guaranteed by the integration, into a coherent whole, of a number of indissociable elements, it no longer depends on the variation of one or other of these factors; the notion of marginal productivity (of labour or of capital) no longer corresponds to any reality ;

- since income can no longer be considered as that which is received in exchange for a contribution to the productive process, the current increase in social benefits could be the caricatural and clumsy foreshadowing of a distributive society towards which we are moving.

The importance of this mutation was perhaps only matched by the mutation in neolithic times, 8 to 10 thousand years ago, when the nomadic populations settled, learnt to use the land systematically to convert solar energy and thus opened up a period where all the great economic (and at the same time social) revolutions of humankind were based on energy. For the first time, the driving forces of development seem to shift away from these fields and, in this sense, we can say that humankind is emerging from the neolithic age. Thus, in the same way that general evolution has gradually freed itself from the weight of organic mass and has progressively focussed on transformations (minor in dimension 
and major in effect) of the nervous system, so does economic evolution seem to free itself from the weight of the material (once the foundations of the latter have been assured, of course) and to move towards the immaterial. The efficiency of the productive apparatus henceforth depends, more and more, on the way it is organised and the paradigm of the future will apparently have to be a paradigm of information. The work of biologists like $\mathrm{H}$. Atlan or H. Laborit, of sociologists like E. Morin and also of the too rare economists and other thinkers whose research is more or less related to economics - such as Ackoff, Boulding, Marschak, Porat, Simon... are laying the foundation stones.

In this direction uncertainty reappears much strengthened: it was absent from Newtonian economics, appeared with Heisenberg but only in the relation between people and things; with thermodynamics it was more deeply rooted in the very behaviour of matter and energy ; and with information we find it in the instrinsic nature of the event : indeed, according to Shannon, we can measure the amount of information contained in the occurrence of an event as a function of the probability that the event will actually occur. The economics of the future will no longer be able to cheat with this reality.

Everything happens as if reality - insofar as our perception allows us to speak of it - disclosed little by little its multiple facets. Far from being mutually exclusive, on the contrary, energy and information show themselves to be indissociable : it is information (in its etymological sense as a structuring activity) which, seen as a difference in potential, allows the production of kinetic energy, without which there can be no activity; and, the other way round, it is energy which, far from the field of thermodynamic equilibrium, provokes phenomena of structuring and organisation which are analysed as creations of form.

An economic system is only productive because it is made up both of energy and information. For a time the first component, because it was the limiting growth factor, hid the role of the second. But once this component seems to have exhausted its effects, when a certain threshold of accumulation has been reached, information takes over.

Uncertainty, which asserts itself more and more throughout this movement, will no doubt for years to come be seen by many a state as that unbearable margin that must, if possible, be reduced, in the name of efficiency; but it will also represent, for every individual, that irreducible flaw in determinism through which a little freedom ineluctably escapes.

\section{BIBLIOGRAPHY}

ATLAN, H. : “L'Organisation Biologique et la Théorie de l'Information ”, Hermann, 1972.

ATLAN, H. : “Entre le Cristal et la Fumée ”, Essai sur l'organisation du vivant, Seuil, 1972.

EIGEN, M.: "Self-Organization of Matter and the Evolution of Biological Macro-Molecules ", in Die Naturwissenschaften, 1971. 
FOERSTER, H. von: "On Self-Organizing Systems and their Environments", in Self-Organizing Systems, Pergamon Press, 1960.

MONOD, J. : “ Le Hasard et la Nécessité ”, Seuil, 1970.

NORA, S., and MINC, A. : "L'Informatisation de la Société ”, Seuil, 1978.

PASSET, R. : “ L'Economique et le Vivant”, Payot, 1979.

PASSET, R. : “La Thermodynamique d'un Monde Vivant: des Structures Dissipatives à l'Economie", Futuribles, December 1980.

PRIGOGINE, I., and STENGERS, I. : “La Nouvelle Alliance ”, Gallimard, 1979.

RUEFF, J. : “ L'Ordre Social ”, 3rd ed., Genin, 1967.

RUEFF, J. : “ Les Dieux et les Rois”, Hachette, 1967.

SCHRÖDINGER, E. : "What is Life?”, Cambridge University Press, 1945. 